\title{
Unidirectionally invisible potentials as local building blocks of all scattering potentials
}

\author{
Ali Mostafazadeh* \\ Departments of Physics and Mathematics, Koç University, Sartyer 34450, Istanbul, Turkey
}

(Received 2 July 2014; published 18 August 2014)

\begin{abstract}
We give a complete solution of the problem of constructing a scattering potential $v(x)$ that possesses scattering properties of one's choice at an arbitrary prescribed wave number. Our solution involves expressing $v(x)$ as the sum of at most six unidirectionally invisible finite-range potentials for which we give explicit formulas. Our results can be employed for designing optical potentials. We discuss its application in modeling threshold lasers, coherent perfect absorbers, and bidirectionally and unidirectionally reflectionless absorbers, amplifiers, and phase shifters.
\end{abstract}

DOI: 10.1103/PhysRevA.90.023833

PACS number(s): 42.25.Bs, 03.65.Nk, 02.30.Zz

Complex scattering potentials in one dimension provide a fertile ground for modeling various active optical systems. Among these are systems displaying spectral singularities [1-4] and unidirectional reflectionlessness and invisibility [5-9]. Spectral singularities correspond to scattering states that behave exactly like zero-width resonances [1]. In optics they give rise to lasing at the threshold gain [10] while their time reversal is responsible for coherent perfect absorption (CPA) or antilasing [11,12]. Unidirectional reflectionlessness and invisibility are also of great interest, because they offer means of realizing one-way linear optical devices [5,7]. The task of designing scattering potentials that support such desirable properties is clearly a problem of basic theoretical and practical importance. In this article we give a complete solution for this problem.

Throughout this investigation, we use the transfer matrix of one-dimensional scattering theory [13] as our main tool. For a scattering potential $v(x)$, the general solution of the Schrödinger equation, $-\psi^{\prime \prime}(x)+v(x) \psi(x)=k^{2} \psi(x)$, or the Helmholtz equation, $\psi^{\prime \prime}(x)+k^{2} \mathfrak{n}(x)^{2} \psi(x)=0$, has the asymptotic form

$$
\psi_{ \pm}(x)=A_{ \pm} e^{i k x}+B_{ \pm} e^{-i k x} \text { for } x \rightarrow \pm \infty,
$$

where $k$ is the wave number, $\mathfrak{n}(x)$ is a refractive index that we can relate to $v(x)$ via $\mathfrak{n}(x)=\sqrt{1-v(x) / k^{2}}$, and $A_{ \pm}$and $B_{ \pm}$are complex coefficients. The transfer matrix of $v(x)$ [and $\mathfrak{n}(x)]$ is the $2 \times 2$ matrix $\mathbf{M}$ satisfying

$$
\mathbf{M}\left[\begin{array}{l}
A_{-} \\
B_{-}
\end{array}\right]=\left[\begin{array}{l}
A_{+} \\
B_{+}
\end{array}\right] .
$$

We can express it in terms of the left and right reflection amplitudes, $R^{l}$ and $R^{r}$, and the transmission amplitude $T$ of $v(x)$ according to [1]

$$
\mathbf{M}=\left[\begin{array}{cc}
T-R^{l} R^{r} / T & R^{r} / T \\
-R^{l} / T & 1 / T
\end{array}\right] .
$$

Recall that the asymptotic scattering solutions of the above Schrödinger and Helmholtz equations have the form

$$
\psi_{l}(x)= \begin{cases}e^{i k x}+R^{l} e^{-i k x} & \text { for } \quad x \rightarrow-\infty, \\ T e^{i k x} & \text { for } \quad x \rightarrow+\infty\end{cases}
$$

\footnotetext{
*amostafazadeh@ku.edu.tr
}

$$
\psi_{r}(x)= \begin{cases}T e^{-i k x} & \text { for } \quad x \rightarrow-\infty \\ e^{-i k x}+R^{r} e^{i k x} & \text { for } \quad x \rightarrow+\infty\end{cases}
$$

and the reflection and transmission coefficients are given by $\left|R^{l / r}\right|^{2}$ and $|T|^{2}$.

We can use Eqs. (1)-(3) to derive the following transformation rules for $R^{l / r}$ and $T$ under the space reflection (parity), $x \stackrel{\mathcal{P}}{\rightarrow}-x$, space translations, $x \stackrel{T_{a}}{\longrightarrow} x-a$, and the time-reversal transformation, $\psi(x) \stackrel{\mathcal{T}}{\rightarrow} \psi(x)^{*}$.

$$
\begin{gathered}
R^{l} \stackrel{\mathcal{P}}{\longrightarrow} R^{r}, \quad R^{r} \stackrel{\mathcal{P}}{\longrightarrow} R^{l}, \quad T \stackrel{\mathcal{P}}{\longrightarrow} T, \\
R^{l} \stackrel{T_{a}}{\longrightarrow} e^{2 i a k} R^{l}, \quad R^{r} \stackrel{T_{a}}{\longrightarrow} e^{-2 i a k} R^{r}, \quad T \stackrel{T_{a}}{\longrightarrow} T, \\
R^{l} \stackrel{\mathcal{T}}{\longrightarrow}-\frac{R^{r *}}{D^{*}}, \quad R^{r} \stackrel{\mathcal{T}}{\longrightarrow}-\frac{R^{l *}}{D^{*}}, \quad T \stackrel{\mathcal{T}}{\longrightarrow} \frac{T^{*}}{D^{*}},
\end{gathered}
$$

where $D:=T^{2}-R^{l} R^{r}$.

We say that $v(x)$ is unidirectionally reflectionless at a wave number $k_{0}$ if for $k=k_{0}, R^{l}=0 \neq R^{r}$, or $R^{r}=0 \neq R^{l}$. We respectively refer to these conditions as "left reflectionlessness" and "right reflectionlessness." Clearly the condition for bidirectional reflectionlessness is $R^{l}=R^{r}=0$. If a potential that is unidirectionally reflectionless at a wave number $k_{0}$ has a unit transmission amplitude, i.e., $T=1$, at this wave number, we call it "unidirectionally invisible" [5]. Similarly, we use "left invisible," "right invisible," and "bidirectionally invisible" to mean that a potential is respectively left reflectionless, right reflectionless, and bidirectionally reflectionless and in addition satisfies $T=1$ at $k=k_{0}$.

In this article we use the properties of the transfer matrix to address the problem of constructing scattering potentials with specific scattering properties at a given real and positive wave number. For future reference we use the term "local inverse scattering" to refer to this problem. We also make use of the notion of the support of a potential $v(x)$ which is the smallest closed interval $I$ outside of which $v(x)$ vanishes. For a pair of potentials, $v_{1}(x)$ and $v_{2}(x)$, with support $I_{1}$ and $I_{2}$, we use the notation " $I_{1} \prec I_{2}$ " to means that $I_{1}$ lies to the left of $I_{2}$, i.e., for all $x_{1} \in I_{1}$ and $x_{2} \in I_{2}$, we have $x_{1} \leqslant x_{2}$. Suppose that $I_{1} \prec I_{2}$ and $v(x)=v_{1}(x)+v_{2}(x)$. Then we can use Eq. (2) to relate $\mathbf{M}$ to the transfer matrices $\mathbf{M}_{i}$ of $v_{i}(x)$ according to $\mathbf{M}=\mathbf{M}_{2} \mathbf{M}_{1}$. This, so-called composition property makes the transfer matrix 
an extremely useful tool in dealing with a variety of physics and engineering problems [13].

If $v(x)$ is a finite-range potential, then $I=[a, b]$, and we can write $v(x)=g(x) \theta(x-a) \theta(b-x)$, where $g(x)$ gives the value of the potential for $x \in[a, b]$, and $\theta(x)$ is the Heaviside step function; $\theta(x):=0$ for $x<0$, and $\theta(x):=1$ for $x \geqslant 0$.

We begin our analysis by considering the local inverse scattering problem for potentials with finite reflection and transmission amplitudes, $R^{l / r}$ and $T$, at the prescribed wave number $k_{0}$. Suppose that we can construct finite-range potentials $v_{ \pm}(x)$ and $v_{0}(x)$ with support $I_{ \pm}$and $I_{0}$ and transfer matrices $\mathbf{M}_{ \pm}$and $\mathbf{M}_{0}$, such that $I_{-} \prec I_{0} \prec I_{+}$and

$$
\begin{gathered}
\mathbf{M}_{-}=\left[\begin{array}{cc}
1 & 0 \\
-R^{l} & 1
\end{array}\right], \quad \mathbf{M}_{+}=\left[\begin{array}{cc}
1 & R^{r} \\
0 & 1
\end{array}\right], \\
\mathbf{M}_{0}=\left[\begin{array}{cc}
T & 0 \\
0 & 1 / T
\end{array}\right],
\end{gathered}
$$

for $k=k_{0}$. Then the transfer matrix of the potential

$$
v(x):=v_{-}(x)+v_{0}(x)+v_{+}(x)
$$

is given by

$$
\mathbf{M}_{+} \mathbf{M}_{0} \mathbf{M}_{-}=\left[\begin{array}{cc}
T-R^{l} R^{r} / T & R^{r} / T \\
-R^{l} / T & 1 / T
\end{array}\right] .
$$

Comparing this relation with Eq. (3), we see that $R^{l}, R^{r}$, and $T$ are respectively the left reflection, right reflection, and transmission amplitudes of $v(x)$ at the wave number $k_{0}$. This reduces the local inverse scattering problem for potentials with finite reflection and transmission amplitudes to the construction of the bidirectionally reflectionless potential $v_{0}(x)$ and the unidirectionally invisible potentials $v_{ \pm}(x)$.

Next, we show that $v_{0}(x)$ can be constructed out of four unidirectionally invisible potentials. Let $\mathcal{R}$ be an arbitrary nonzero complex number, and for each $j \in\{1,2,3,4\}, v_{j}(x)$ is a finite-range unidirectionally invisible potential with support $I_{j}$ and reflection amplitudes $R_{j}^{l / r}$ at wave number $k_{0}$ such that $I_{1} \prec I_{2} \prec I_{3} \prec I_{4}, R_{1}^{r}=R_{2}^{l}=R_{3}^{r}=R_{4}^{l}=0$, and

$$
R_{1}^{l}=-\mathcal{R} T, \quad R_{2}^{r}=\frac{T-1}{\mathcal{R} T}, \quad R_{3}^{l}=\mathcal{R}, \quad R_{4}^{r}=\frac{1-T}{\mathcal{R}} .
$$

Then $v_{1}(x)$ and $v_{3}(x)$ are right invisible while $v_{2}(x)$ and $v_{4}(x)$ are left invisible, and the transfer matrix $\mathbf{M}_{j}$ of $v_{j}(x)$ at $k_{0}$ has the form

$$
\begin{array}{ll}
\mathbf{M}_{1}=\left[\begin{array}{cc}
1 & 0 \\
\mathcal{R} T & 1
\end{array}\right], & \mathbf{M}_{2}=\left[\begin{array}{cc}
1 & (T-1) / \mathcal{R} T \\
0 & 1
\end{array}\right], \\
\mathbf{M}_{3}=\left[\begin{array}{cc}
1 & 0 \\
-\mathcal{R} & 1
\end{array}\right], & \mathbf{M}_{4}=\left[\begin{array}{cc}
1 & (1-T) / \mathcal{R} \\
0 & 1
\end{array}\right] .
\end{array}
$$

It is an easy exercise to show that for $k=k_{0}$ the transfer matrix of the potential $\sum_{j=1}^{4} v_{j}(x)$ is given by

$$
\mathbf{M}_{4} \mathbf{M}_{3} \mathbf{M}_{2} \mathbf{M}_{1}=\left[\begin{array}{cc}
T & 0 \\
0 & 1 / T
\end{array}\right] .
$$

This shows that we can take

$$
v_{0}(x)=v_{1}(x)+v_{2}(x)+v_{3}(x)+v_{4}(x),
$$

i.e., we can construct a bidirectionally reflectionless potential by assembling four unidirectionally invisible potentials.

Next, we examine the problem of constructing a scattering potential whose reflection and transmission amplitudes diverge at a prescribed real and positive wave number $k_{0}$. Let $w_{ \pm}(x)$ be a pair of scattering potentials with support $J_{ \pm}$such that for the wave number $k_{0}, w_{ \pm}(x)$ has the same transfer matrix as $v_{ \pm}(x)$, but $J_{+} \prec J_{-}$. Then, in view of Eq. (7), the transfer matrix of the potential

$$
w(x):=w_{-}(x)+w_{+}(x)
$$

at $k_{0}$ is given by

$$
\mathbf{M}_{-} \mathbf{M}_{+}=\left[\begin{array}{cc}
1 & R^{r} \\
-R^{l} & 1-R^{l} R^{r}
\end{array}\right] .
$$

Comparing this relation with Eq. (3), we see that the transmission amplitude of $w(x)$ diverges whenever

$$
R^{l} R^{r}=1
$$

If this condition holds, the reflection amplitudes of $w(x)$ also diverge for $k=k_{0}, k_{0}^{2}$ is a spectral singularity [1] of the potential $w(x)$, and $w(x)^{*}$ displays CPA at $k_{0}$ [12].

The arguments we have so far presented show that we can realize any kind of scattering effect at a given wave number using certain finite-range potentials that are the sum of up to six unidirectionally invisible potentials. This solves the local inverse scattering problem for general potentials provided that we can construct the constituent unidirectionally invisible potentials, namely, $v_{ \pm}(x), v_{j}(x)$, and $w_{ \pm}(x)$. In the following we achieve this by introducing a particular family of finite-range unidirectionally invisible potentials.

First, we examine the case of right-invisible potentials, i.e., for a given nonzero complex number $R$, we construct a scattering potential whose right reflection, left reflection, and transmission amplitudes at a prescribed wave number $k_{0}$ are respectively given by $R^{r}=0, R^{l}=R$, and $T=1$.

Consider the finite-range potentials of the form

$$
\begin{aligned}
v_{\alpha, n}(x) & :=k^{2} f_{\alpha}(x) \theta(x) \theta\left(L_{n}-x\right), \\
f_{\alpha}(x) & :=\frac{-8 \alpha\left(3-2 e^{2 i k_{0} x}\right)}{e^{4 i k_{0} x}+\alpha\left(1-e^{2 i k_{0} x}\right)^{2}},
\end{aligned}
$$

where $\alpha$ and $n$ take real and positive integer values, respectively, and $L_{n}:=\pi n / k_{0}$. As we show in the Appendix, $v_{\alpha, n}(x)$ is right invisible at $k=k_{0}$, and for $\alpha>-1 / 4$ its left reflection amplitude at $k_{0}$ has the form

$$
R^{l}=R_{\alpha, n}^{l}:=\frac{-8 \pi i n \alpha}{(\alpha+1)^{3}} .
$$

Because $|\alpha|$ and $n$ can respectively take arbitrarily small and large positive values, we can always choose them such that $8 \pi n \alpha /(\alpha+1)^{3}=|R|$. Let $\varphi \in[0,2 \pi)$ denote the phase angle of $R$, so that $R=|R| e^{i \varphi}$. Then Eq. (5) implies that for every integer $m$, the translation $x \rightarrow x+d$ with

$$
d:=\frac{(4 m-1) \pi-2 \varphi}{4 k_{0}},
$$


maps $v_{\alpha, n}(x)$ to the potential

$$
v_{R}^{r}(x):=v_{\alpha, n}(x+d),
$$

which is right invisible at $k=k_{0}$, and its left reflection amplitude at this wave number coincides with $R$. This completes the solution of the local inverse scattering problem for rightinvisible potentials. Because every left-invisible potential can be written as the complex conjugate of a right-invisible potential [see Eq. (6)], the above construction solves the local inverse scattering problem for all unidirectionally invisible potentials.

$v_{R}^{r}(x)$ and $v_{R}^{r}(x)^{*}$ are finite-range potentials with support $\left[-d, L_{n}-d\right]$. Because $m$ is an arbitrary integer, we can choose it so that the support of these potentials lie to the left or right of any given finite interval. This shows that by making appropriate choices for $\alpha, n, \varphi$, and $m$, we can use $v_{R}^{r}(x)$ and $v_{R}^{r}(x)^{*}$ to obtain an explicit realization of the potentials $v_{ \pm}(x)$, $v_{j}(x)$, and $w_{ \pm}(x)$ that appear in Eqs. (9), (12), and (13). This completes our solution of the local inverse scattering problem for general scattering potentials.

As a concrete example, let us identify the potential $w_{+}(x)$ with $v_{\alpha, n}\left(x+L_{n}\right)^{*}$, where $v_{\alpha, n}(x)$ is given by Eq. (15), $\alpha>-1 / 4$, and $n$ is a positive integer. Then the support of $w_{+}(x)$ is $\left[-L_{n}, 0\right]$. According to Eq. $(5), w_{+}(x)$ has the same transmission amplitude as $v_{\alpha, n}(x)^{*}$, and its left (right) reflection amplitude differs from that of $v_{\alpha, n}(x)^{*}$ by a phase factor $e^{-2 i k L_{n}}\left(e^{2 i k L_{n}}\right)$. But because $k L_{n}$ is an integer multiple of $\pi, e^{ \pm 2 i k L_{n}}=1$. This together with Eqs. (6) and (17) implies that at $k=k_{0}, T=1, R^{l}=0$, and

$$
R^{r}=-R_{\alpha, n}^{l *}=R_{\alpha, n}^{l}=\frac{-8 \pi i n \alpha}{(\alpha+1)^{3}} .
$$

Next, we identify $w_{-}(x)$ with $v_{\beta, m}(x)$ where $\beta>-1 / 4$ and $m$ is a positive integer. Then $w_{-}(x)$ is a potential with support $\left[0, L_{m}\right]$ that is right invisible at $k=k_{0}$, and in light of Eq. (17) its left-reflection amplitude at this wave number is given by

$$
R^{l}=R_{\beta, m}^{l}=\frac{-8 \pi i \beta m}{(\beta+1)^{3}} .
$$

Substituting this relation and Eq. (20) in Eq. (14) gives

$$
(\alpha+1)^{3}(\beta+1)^{3}+64 \pi^{2} m n \alpha \beta=0 .
$$

It turns out that for each nonzero $\alpha$ greater than $-1 / 4$ and positive integers $m$ and $n$, this equation can be solved to find values for $\beta$ that is greater than $-1 / 4$. In particular, for situations where $|\alpha| \ll 1$, there is a single value of $\beta$ satisfying $|\beta| \ll 1$. For this value of $\beta$, the above construction works and the potential (13) has a spectral singularity at the wave number $k_{0}$. In view of Eqs. (15) and (16) the refractive index $\mathfrak{n}(x)$ associated with this potential satisfies

$$
\mathfrak{n}^{2}(x)= \begin{cases}1+\frac{8 \alpha\left(3-2 e^{-2 i k_{0} x}\right)}{e^{-4 i k_{0} x}+\alpha\left(1-e^{-2 i k_{0} x}\right)^{2}} & \text { for } x \in\left[-L_{n}, 0\right), \\ 1+\frac{8 \beta\left(3-2 e^{2 i k_{0} x}\right)}{e^{4 i k_{0} x}+\beta\left(1-e^{2 i k_{0} x}\right)^{2}} & \text { for } x \in\left[0, L_{m}\right], \\ 1 & \text { otherwise. }\end{cases}
$$

An optical system given by the complex conjugate of this refractive index profile displays CPA at $k=k_{0}$.

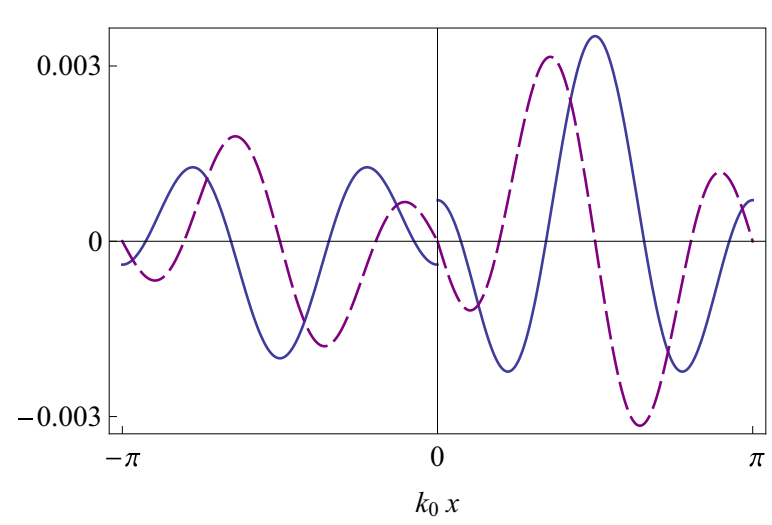

FIG. 1. (Color online) Graphs of real part of of $\mathfrak{n}(x)-1$ (solid blue curve) and the imaginary part of $\mathfrak{n}(x)$ (dashed purple curve) for $\mathfrak{n}(x)$ given by Eq. (23) with $\alpha=-10^{-4}, \beta=1.759 \times 10^{-4}$, and $\left|k_{0} x\right| \leqslant \pi$.

For example, let us take $\alpha=-10^{-4}$ and $m=n=300$. Then Eq. (22) gives $\beta=1.759 \times 10^{-4}$. These numerical values are consistent with the fact that in typical optical applications, $\left|\mathfrak{n}_{\alpha, n}-1\right|$ and $\left|\mathfrak{n}_{\beta, m}-1\right|$ are at most of the order of $10^{-3}$. Figure 1 shows the plots of real and imaginary parts of $\mathfrak{n}(x)-$ 1 for these choices of $\alpha$ and $\beta$, and $\left|k_{0} x\right| \leqslant \pi$. If we take $k_{0}=$ $2 \pi(\mu \mathrm{m})^{-1}$ (wavelength $\left.=1 \mu \mathrm{m}\right), L_{m}=L_{n}=300 \pi / k_{0}=$ $150 \mu \mathrm{m}$. Therefore, this setup corresponds to an infinite planar slab of inhomogeneous optically active material of thickness $300 \mu \mathrm{m}$. Substituting these numerical values for $\alpha, \beta, m$, and $n$ in Eqs. (20) and (21), we find $R^{l}=0.754 i$ and $R^{r}=-1.323 i$. These match the numerical calculation of these quantities. Figure 2 shows the logarithmic plots of the reflection and transmission coefficients for this model. The extremely sharp peak at $k=k_{0}$ is clear evidence of the presence of a spectral singularity.

The ability to use finite-range unidirectionally invisible potentials to realize arbitrary scattering effects provides a powerful method for designing optical potentials. For example,

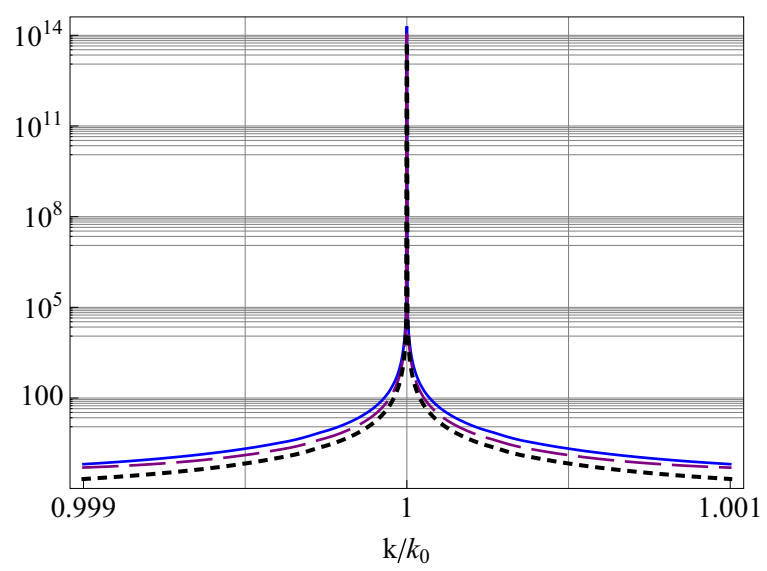

FIG. 2. (Color online) Graphs of the reflection and transmission coefficients $\left|R^{l}\right|^{2}$ (blue full curve), $\left|R^{r}\right|^{2}$ (black dotted curve) and $|T|^{2}$ (purple dashed curve) as functions of $k / k_{0}$ for the refractive index profile given by Eq. (23) with $\alpha=-10^{-4}$ and $\beta=1.759 \times 10^{-4}$. The sharp peak at $k=k_{0}$ is an obvious signature of a spectral singularity. 
consider the construction of the bidirectionally reflectionless potential $v_{0}(x)$ in terms of the unidirectionally invisible potentials $v_{j}(x)$. Suppose that we take

$$
\begin{gathered}
R_{1}^{l}=-\epsilon, \quad R_{2}^{r}=e^{i \theta} \epsilon-\epsilon^{-1}, \\
R_{3}^{l}=e^{-i \theta} \epsilon^{-1}, \quad R_{4}^{r}=e^{i \theta} \epsilon-e^{2 i \theta} \epsilon^{3},
\end{gathered}
$$

where $\epsilon$ is a positive real number and $\theta \in[0,2 \pi)$. Then the potential Eq. (12) is a bidirectionally reflectionless potential with transmission amplitude $T=\epsilon^{2} e^{i \theta}$. We can use the potential Eq. (19) and its complex conjugate to realize $v_{j}(x)$ for arbitrary values of $\epsilon$. In particular, for $\epsilon<1, v_{0}(x)$ models a bidirectionally reflectionless absorber that operates at the wave number $k_{0}$ and induces a specific phase shift in the transmitted wave. Notice that for $\epsilon \ll 1,\left|R_{4}^{r}\right| \approx\left|R_{1}^{l}\right|=\epsilon$ and $\left|R_{2}^{r}\right| \approx$ $\left|R_{3}^{l}\right|=\epsilon^{-1}$. Therefore, we obtain a quadratic absorption effect corresponding to $|T|=\epsilon^{2}$ using unidirectionally invisible amplifiers, $v_{1}(x)$ and $v_{4}(x)$, and unidirectionally invisible absorbers, $v_{2}(x)$ and $v_{3}(x)$, whose strength is linear in $\epsilon$.

Clearly, for $\epsilon=1$, the above system operators as a bidirectionally reflectionless phase shifter, and for $\epsilon>1$ it serves as a bidirectionally reflectionless transmission amplifier that produces a specific phase shift. We can also construct unidirectionally reflectionless absorbers, amplifiers, and phase shifters. This follows from the observation that we can construct unidirectionally reflectionless potentials by adding to $v_{0}(x)$ a finite-range unidirectionally invisible potential $u(x)$ whose support $J$ lies to the left or right of $I_{0}$. Specifically, if $u(x)$ is left (right) invisible and $I_{0} \prec J\left(J \prec I_{0}\right), v_{0}(x)+u(x)$ is left (right) reflectionless.

For example suppose that we wish to use the above construction to obtain a bidirectionally reflectionless amplifier that doubles the intensity of the incident wave $\left(|T|^{2}=2\right)$ and induces a $\pi / 2$-phase shift at the wavelength $1 \mu \mathrm{m}$ $\left(k_{0}=2 \pi / \mu \mathrm{m}\right)$ upon transmission. This corresponds to setting $\epsilon=2^{1 / 4}$ and $\theta=\pi / 2$ in Eqs. (24) and (25). A potential $v_{0}(x)$

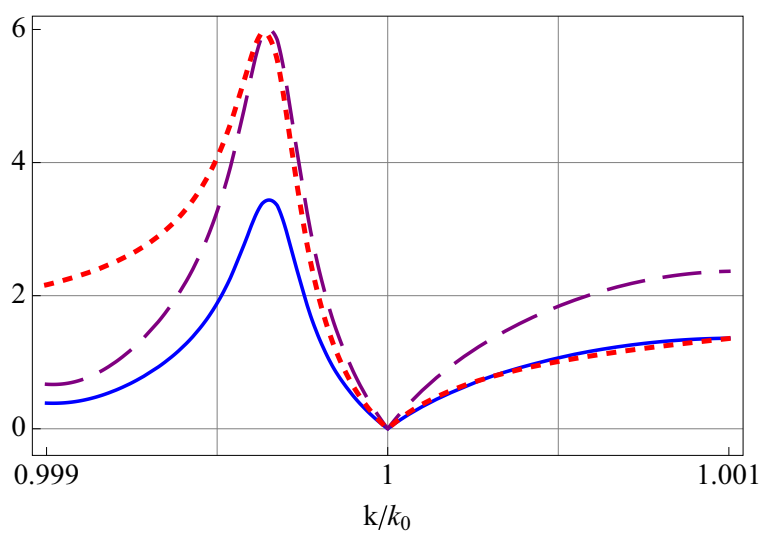

FIG. 3. (Color online) Graphs of $\left|R^{l}\right|$ (solid blue curve) and $\left|R^{r}\right|$ (dashed purple curve), and $|T-\sqrt{2} i|$ (dotted red curve) as functions of $k / k_{0}$ for the potential given by Eqs. (12) and (26), and $k_{0}=$ $2 \pi / \mu \mathrm{m}$. The fact that all of these quantities vanish for $k=k_{0}$ shows that this potential models a bidirectionally reflectionless amplifier that doubles the intensity of the incident wave upon transmission and induces a $\pi / 2$ phase. that achieves this goal is given by Eq. (12) where

$$
v_{j}(x):=\left\{\begin{array}{lll}
v_{\alpha_{j}, n}\left(x+d_{j}\right) & \text { for } & j=1,3, \\
v_{\alpha_{j}, n}\left(x+d_{j}\right)^{*} & \text { for } & j=2,4,
\end{array}\right.
$$

$n=300$, so that $L_{n}=150 \mu \mathrm{m}$, and

$$
\begin{array}{ll}
\alpha_{1}=1.57798 \times 10^{-4}, & d_{1}=300.625 \mu \mathrm{m}, \\
\alpha_{2}=1.93283 \times 10^{-4}, & d_{2}=150.299 \mu \mathrm{m}, \\
\alpha_{3}=1.11565 \times 10^{-4}, & d_{3}=0.00000 \mu \mathrm{m}, \\
\alpha_{4}=2.73409 \times 10^{-4}, & d_{4}=-150.326 \mu \mathrm{m} .
\end{array}
$$

In particular, the support $I_{j}$ of $v_{j}(x)$ that is given by $\left[-d_{j}, L_{n}-d_{j}\right]$ fulfills the requirement $I_{1} \prec I_{2} \prec I_{3} \prec I_{4}$. $v_{0}(x)$ corresponds to a particular gain-loss profile that is confined in a planar slab of thickness $600.951 \mu \mathrm{m}$. Figure 3 shows the plots of $\left|R^{l}\right|,\left|R^{r}\right|$, and $|T-\sqrt{2} i|$ for this system. The vanishing of all these quantities at $k=k_{0}$ is a graphical (numerical) confirmation of our analytical results.

In conclusion, we have revealed an intriguing property of unidirectionally invisible potentials, namely, that using at most six such potentials we can realize any scattering effects at any wave number. This solution allows for designing a variety of optical potentials that can model threshold lasers, antilasers, and bidirectionally and unidirectionally reflectionless absorbers, amplifiers, and phase shifters. The main disadvantage of our construction is that it yields potentials that realize the desired scattering properties only at a single wave number. However, it also implies that these properties are essentially preserved in the spectral bands in which the constituent unidirectionally invisible potentials retain their scattering features. This reduces the general inverse scattering problem for arbitrary real or complex scattering potentials to constructing broadband unidirectionally invisible potentials. The study of this kind of potential can be conducted using the standard inverse scattering theory [14] which is typically difficult to apply, for it involves solving certain integral equations. In this context, it might be useful to note the possibility of constructing broadband unidirectionally reflectionless bilayer slabs that operate in spectral bands as wide as $100 \mathrm{~nm}$ [8]. Finally, we note that our approach allows us to construct potentials that display a particular scattering effect at more than one wave number. This requires using constituent unidirectionally invisible potentials that have the appropriate properties at these wave numbers. The problem of constructing finite-range potentials displaying perturbative unidirectional invisibility at finitely or infinitely many wave numbers is treated in Ref. [9].

This work was supported by the Scientific and Technological Research Council of Turkey (TÜBITAK) in the framework of Project No. 112T951, and by the Turkish Academy of Sciences (TÜBA).

\section{APPENDIX}

In Ref. [15] we show that the reflection and transmission amplitudes of a finite-range potential $v(x)$ with support $[0, L]$ 
are given by

$$
\begin{gathered}
R^{l}=-\int_{1}^{z_{+}} d z \frac{S^{\prime \prime}(z)}{S(z) S^{\prime}(z)^{2}}, \\
R^{r}=\frac{S\left(z_{+}\right)}{S^{\prime}\left(z_{+}\right)}-z_{+}, \quad T=\frac{1}{S^{\prime}\left(z_{+}\right)},
\end{gathered}
$$

where $z:=e^{-2 i k x}, z_{+}:=e^{-2 i k L}, S(z)$ is the solution of the initial-value problem

$$
z^{2} S^{\prime \prime}(z)+\left[\frac{\breve{v}(z)}{4 k^{2}}\right] S(z)=0, \quad S(1)=S^{\prime}(1)=1,
$$

$\check{v}(z):=v(i \ln z /(2 k))=v(x)$, and the integral in Eq. (A1) is to be evaluated along the clockwise-oriented circular arc: $\left\{e^{-2 i t} \mid t \in[0, k L]\right\}$. In particular, whenever $k L=\pi n$ for some positive integer $n, z_{+}=1$, and the integral in Eq. (A1) becomes a contour integral that is to be evaluated along the clockwise-oriented contour $\gamma_{n}:=\left\{e^{-i t} \mid t \in[0,2 \pi n]\right\}$, i.e., the $n$-fold covering of the unit circle $\gamma_{1}$. In this case, $R^{r}=0$, $T=1$, and $v(x)$ is a right-invisible potential provided that $R^{l} \neq 0$. The latter relation holds whenever the integrand in Eq. (A1) has poles inside $\gamma_{1}$, and the sum of the residues of the integrand at these poles is nonzero.

The potential $v_{\alpha, n}(x)$ introduced in Eq. (15) corresponds to setting $k=k_{0}, L=n \pi / k_{0}$, and $S(z)=z\left[\alpha(z-1)^{2}+1\right]$. This choice for $S(z)$ fulfills Eq. (A3) if we set $v(x)=v_{\alpha, n}(x)$. It is also easy to check that this potential is smooth on its support except for $\alpha=-1 / 4$ where it develops a singularity at $x=(2 \ell+1) \pi / 2 k$ for integer $\ell$. Furthermore, for $\alpha>-1 / 4$, the integrand in Eq. (A1) has a single pole lying inside $\gamma_{1}$ (namely, $z=0$ ). In this case, we can easily use the residue theorem to evaluate the right-hand side of Eq. (A1). This results in Eq. (17).
[1] A. Mostafazadeh, Phys. Rev. Lett. 102, 220402 (2009); 110, 260402 (2013).

[2] A. Mostafazadeh, Phys. Rev. A 80, 032711 (2009); A. Mostafazadeh and M. Sarısaman, Phys. Lett. A 375, 3387 (2011); Proc. R. Soc. A 468, 3224 (2012); Phys. Rev. A 87, 063834 (2013); 88, 033810 (2013); A. Mostafazadeh and S. Rostamzadeh, ibid. 86, 022103 (2012).

[3] S. Longhi, Phys. Rev. B 80, 165125 (2009); Phys. Rev. A 81, 022102 (2010); Z. Ahmed, J. Phys. A 42, 472005 (2009); 45, 032004 (2012).

[4] F. Correa and M. S. Plyushchay, Phys. Rev. D 86, 085028 (2012); L. Chaos-Cador and G. Garcia-Calderon, Phys. Rev. A 87, 042114 (2013); A. Sinha and R. Roychoudhury, J. Math. Phys. 54, 112106 (2013); X. Liu, S. Dutta Gupta, and G. S. Agarwal, Phys. Rev. A 89, 013824 (2014).

[5] Z. Lin, H. Ramezani, T. Eichelkraut, T. Kottos, H. Cao, and D. N. Christodoulides, Phys. Rev. Lett. 106, 213901 (2011).

[6] L. Poladian, Phys. Rev. E 54, 2963 (1996); M. Greenberg and M. Orenstein, Opt. Lett. 29, 451 (2004); M. Kulishov, J. M. Laniel, N. Belanger, J. Azana, and D. V. Plant, Opt. Express 13, 3068 (2005); S. Longhi, J. Phys. A 44, 485302 (2011); H. F.
Jones, ibid. 45, 135306 (2012); R. Uzdin and N. Moiseyev, Phys. Rev. A 85, 031804 (2012); A. Regensburger, C. Bersch, M. A. Miri, G. Onishchukov, D. N. Christodoulides, and U. Peschel, Nature 488, 167 (2012).

[7] L. Feng, Y.-L. Xu, W. S. Fegasolli, M.-H. Lu, J. E. B. Oliveira, V. R. Almeida, Y.-F. Chen, and A. Scherer, Nat. Mater. 12, 108 (2013); X. Yin and X. Zhang, ibid. 12, 175 (2013).

[8] A. Mostafazadeh, Phys. Rev. A 87, 012103 (2013).

[9] A. Mostafazadeh, Phys. Rev. A 89, 012709 (2014).

[10] A. Mostafazadeh, Phys. Rev. A 83, 045801 (2011); 87, 063838 (2013).

[11] Y. D. Chong, L. Ge, H. Cao, and A. D. Stone, Phys. Rev. Lett. 105, 053901 (2010); W. Wan, Y. Chong, L. Ge, H. Noh, A. D. Stone, and H. Cao, Science 331, 889 (2011).

[12] S. Longhi, Phys. Rev. A 82, 031801 (2010); 83, 055804 (2011); Phys. Rev. Lett. 107, 033901 (2011).

[13] L. L. Sánchez-Soto, J. J. Monzóna, A. G. Barriuso, and J. F. Cariñena, Phys. Rep. 513, 191 (2012).

[14] K. Chadan and P. C. Sabatier, Inverse Problems in Quantum Scattering Theory (Springer, New York, 1989).

[15] A. Mostafazadeh, Ann. Phys. (NY) 341, 77 (2014). 